Bull. Austral. Math. Soc.

VOL. $74(2006) \quad[247-262]$

\title{
ON ISOMETRIC ACTIONS
}

\author{
S.B. MULAY
}

To a cardinal $k \geqslant 2$, we associate a simply-connected polyhedral surface $\Sigma_{k}$ endowed with a bounded metric $d_{k}$ such that every group of cardinality $k$ has an isometric, properly discontinuous action on $\left(\Sigma_{k}, d_{k}\right)$. If $\aleph_{0} \leqslant k \leqslant 2^{\aleph_{0}}$ and $G$ is a group of cardinality $k$, then we extend $\left(\Sigma_{k}, d_{k}\right)$ to a simply-connected bounded metric space $\left(M_{G}, d_{G}\right)$ such that the action of $G$ extends to an isometric, properly discontinuous action on $\left(M_{G}, d_{G}\right)$ and $G$ is the full isometry-group of $\left(M_{G}, d_{G}\right)$.

\section{INTRODUCTION}

Let $G$ be a group. Suppose we are given an isometric action of $G$ on a metric space $M$. By an isometric action we mean that each element of $G$ acts not merely as a homeomorphism but as an isometry of $M$. In this situation can we always extend $M$ nicely to a larger metric space so that $G$ is realised as the full isometry group of the extension? Here nicely means, on the one hand, preserving the properties of action such as freeness, discreteness, discontinuity et cetera and, on the other hand, preserving boundedness, connectedness properties et cetera of $M$. In Section 3 of this article we have formulated one possible definition of what can be regarded as a nice extension.

The main aim of this article is to provide a partial answer to the above question. Our construction of such a desired extension $\mathcal{M}$ of $M$ is under the restrictions that $M$ is a connected, bounded metric space of cardinality $\leqslant 2^{\aleph_{0}}$ and $G$ is an infinite group of cardinality $\leqslant 2^{\aleph_{0}}$. Most groups of traditional interest (for example, the linear groups) do fall in this restricted class. But, there are a number of interesting isometric actions for which the underlying metric is un-bounded. Therefore it is desirable to remove, in some manner, our imposed restriction of boundedness. Also, the case of a finite group $G$ acting isometrically on a nontrivial metric space $M$ remains outside our consideration. We construct $\mathcal{M}$ by attaching spikes (or hair) to $M$; for this reason we have termed it as a comb-extension. Consequently, $M$ can be recovered as a deformation retract of its enlargement $\mathcal{M}$. These spikes are of equal length but their relative positioning (or manner of attachment) depends on $G$ as well as its given action on $M$.

Consider a nontrivial group $G$ (of arbitrary cardinality) as a discrete topological space. Let $X$ be a cone over $G$ having vertex $O$. The left-multiplication action of $G$ on

Received 18th April, 2006

Copyright Clearance Centre, Inc. Serial-fee code: 0004-9727/06 \$A2.00+0.00. 
itself has a canonical extension to an action on $X$ such that $O$ is the only point with non-trivial stabiliser. There is a natural path metric on $X$ with respect to which this action of $G$ is isometric. We deform $X^{3}-\{(O, O, O)\}$ to a simply-connected surface $\Sigma$ which is a patchwork of triangles (2-simplices) and such that the coordinatewise action of $G$ on $X^{3}-\{(O, O, O)\}$ leaves $\Sigma$ invariant. This way we get an isometric, properly discontinuous action of $G$ on $\Sigma$. Our metric spaces $X, \Sigma$ depend only on the cardinality of $G$. If $G$ is countable, $\Sigma$ is separable and admits a topological embedding in $\mathbb{R}^{6}$.

When the order of $G$ does not exceed that of the real numbers, we construct a bounded metric space $\left(N_{G}, d_{G}\right)$ with an isometric action of $G$ satisfying the following three key requirements: the distance between any pair of distinct points is bounded (below) away from zero, $G$ is the exact full group of isometries of $\left(N_{G}, d_{G}\right)$ and the action of $G$ is properly discontinuous. Our cardinality restriction is needed only in this construction. Further, if $G$ is infinite, a comb-extension of $\Sigma$ by means of $\left(N_{G}, d_{G}\right)$ provides a simply-connected, bounded metric space having $G$ as its full isometry-group and on which, the action of $G$ remains properly-discontinuous.

En route we also show that a finite group can be realised as the full isometry-group of a finite metric space. This is well-known (see [1]) but the construction employed in [1] is of different nature. The main theorem of [1] shows that any finite group $G$ can be realised as the full isometry-group of a Riemannian sphere; however, the action of $G$ resulting from this construction is rarely free of fixed points. In the last section of this article we pose some problems closely related to our explorations. Concerning these, very little appears to be known. We are indeed aware of the connection between group-actions and Cayley-graphs, but how advantageous it is in the present context, remains to be seen.

\section{GRAPH-AUTOMORPHISMS AND ISOMETRIES}

The set of all nonempty, proper subsets of a set $K$ is called the restricted power-set of $K$ and we denote it by $\pi(K)$. By the restricted power-set graph of $K$ we mean the graph whose vertex-set is $\pi(K)$ and there is an undirected edge between $A, B \in \pi(K)$ whenever $A \cup B=K$. Since there is hardly any possibility of confusion, the subgraph of the restricted power-set graph of $K$ with an underlying set of vertices $L \subseteq \pi(K)$ is also denoted by $L$. The group of permutations of $K$ is denoted by $S(K)$. The induced action of $S(K)$ on $\pi(K)$ is given by: $A^{\sigma}:=\sigma(A)$ for all $(A, \sigma) \in \pi(K) \times S(K)$.

By the canonical metric of a connected graph we mean the metric on its vertex-set obtained by declaring each edge to have length 1 and where the distance between any two vertices is defined to be the length of a shortest edge-path connecting them. It is easy to verify that the diameter of $\pi(K)$, with respect to its canonical metric, is at most 3 (see the proof of assertion (i) of Lemma 1 below).

Let $G$ be a non-trivial group and let $X:=G \times\{0,1\}$. The action of $G$ on $X$ by left-multiplication in the first coordinate turns $X$ into a $G$-set. From this we get the 
induced action of $G$ on $\pi(X)$. In the rest of this article $\pi(X)$ is regarded as a $G$-set via this action. Given a subset $A$ of $X$ and an index $i \in\{0,1\}$, we let $A_{i}:=A \cap G \times\{i\}$. In the following $G$ and $X$ remain fixed.

Lemma 0 . An element $A \in \pi(X)$ has non-trivial stabiliser in $G$ if and only if each $A_{i}$ is a union of right-cosets of some non-trivial cyclic subgroup of $G$.

Proof: Let $g$ be a non-identity element of the stabiliser of $A$ in $G$. Clearly $g A_{i}$ $=g^{-1} A_{i}=A_{i}$ for each $i$. Without loss we regard each $A_{i}$ as a subset of $G$. Given an $A_{i}$ which is either the empty or the improper subset of $G$, our assertion is obviously true. Say $A_{0}$ is a nonempty proper subset of $G$. Suppose $\langle g\rangle x$ is a coset which is not contained in $A_{0}$ but $A_{0} \cap\langle g\rangle x$ is nonempty. Pick $a$ in $A_{0} \cap\langle g\rangle x$ and $b \in\langle g\rangle x \backslash A$. Now $a:=g^{p} x$ and $b:=g^{q} x$ for some integers $p, q$. So $g^{(q-p)} a=b$. Since $g$ stabilises $A$, the element $g^{q-p} a$ is in $A_{0}$. This contradicts the choice of $b$. Hence a right coset of $\langle g\rangle$ is either contained in $A_{0}$ or it is disjoint from $A_{0}$. In other words, $A_{0}$ is a union of right cosets of the nontrivial cyclic group $\langle g\rangle$. The converse is straightforward.

Definition: A sub-set $W$ of $\pi(X)$ is called nice if

(1) $W$ is closed under complementation,

(2) $W$ contains the singleton subsets of $X$,

(3) $W$ contains the subsets of $X$ of the form $\{(g, 0),(h, 1)\}$,

(4) $\operatorname{stab}_{G}(A)$ (the stabiliser of $A$ in $G$ ) is trivial for each $A \in W$ and

(5) for all $(g, A) \in G \times W$ the set $g A$ is in $W$.

EXAMPLES.

(e1) Let $Z$ denote the subset of $\pi(X)$ consisting of those $A \in \pi(X)$ whose stabiliser in $G$ is the trivial subgroup.

(e2) Let $K \subseteq \pi(X)$ consist of the singleton subsets of $X$ together with the subsets (of $X$ ) of the form $\{(g, 0),(h, 1)\}$. Let $Z_{0}$ be the set of those $A \in \pi(X)$ for which either $A \in K$ or $X-A \in K$.

(e3) For an $r$ which is either an integer $\geqslant 3$ or $\infty$, let $Z_{r}(X)$ denote the subset of $Z$ which consists of all $A \in Z$ such that either $\operatorname{card}(A)<r$ or $\operatorname{card}(X$ $-A)<r$.

REMARKS.

1. $Z_{0} \subseteq Z_{r}(X) \subseteq Z_{r+1}(X) \subseteq Z_{\infty}(X)$ for all $r \geqslant 3$.

2. Sets $Z, Z_{0}$ and $Z_{r}$, for all $3 \leqslant r \leqslant \infty$, are nice.

3. If $W$ is nice, then $Z_{0} \subseteq W$.

4. If $G$ is infinite, then $\operatorname{card}\left(Z_{0}\right)=\operatorname{card}\left(Z_{r}\right)=\operatorname{card}(G)$ for all $3 \leqslant r \leqslant \infty$.

5. Thanks to Lemma 0 , we have an injective function $\gamma: \pi(G) \rightarrow Z$ given by

$$
\gamma(T):=(T \times 0) \cup\{(e, 1)\}
$$


where $e$ stands for the identity element of $G$. If $G$ is infinite, then from the existence of $\gamma$ we see that $Z$ and $\pi(X)$ have the same cardinality. In particular if $G$ is infinite countable, then $\operatorname{card}(Z)=2^{\mathrm{N}_{0}}$ (the cardinality of real numbers).

LEMmA 1. Let $W$ be a nice subset of $\pi(X)$. Then the following holds.

(i) $W$ is a connected graph of diameter at most 3.

(ii) If $\delta$ denotes the canonical metric of the graph $W$, then it coincides with the restriction of the canonical metric of $\pi(X)$ to $W$.

(iii) $W$ does not have vertices of degree 2. Singleton subsets of $X$ are the only vertices of degree 1 in $W$.

(iv) $W$ is a union of $\pi(X)$-orbits of $G$.

(v) Let Aut $(W)$ denote the group of graph-automorphisms of $W$. Then $G$ is, in a natural way, a subgroup of $\operatorname{Aut}(W)$.

(vi) $\operatorname{Aut}(W)$ is the group of isometries of $(W, \delta)$.

Proof: Suppose $A, B$ are two distinct vertices of $W$. If $A$ is comparable to $B$ with respect to containment, say $A \subset B$, then $A$ to $X-A$ to $B$ is an edge-path of length 2 from $A$ to $B$. Otherwise, picking $a \in A \backslash B$ and $b \in B \backslash A$, we get an edge-path from $A$ to $X-\{a\}$ to $X-\{b\}$ to $B$ which is in $W$ and has length 3. Thus (i) holds.

Now $A$ is at distance 1 from $B$ in $\pi(X)$ if and only if this is so in $W$. Suppose $A \cup B \neq X$ but $A$ to $T$ to $B$ is an edge-path in $\pi(X)$. Then $T$ contains $X-(A \cap B)$. So $A \cap B$ is non-empty. Pick $x \in A \cap B$. Then $A$ to $X-\{x\}$ to $B$ is an edge-path of length 2 in $W$. This establishes (ii).

Consider a vertex $A$ of $W$. If $A$ has at least 2 distinct elements $a, b$ then $A$ is adjacent to three distinct vertices $X-\{a\}, X-\{b\}$ and $X-A$ of $W$. If $A$ is singleton, then its degree is clearly 1 . This proves (iii).

Assertion (iv) is simply a re-statement of no.(5) (in the definition of niceness). The action of $G$ on $W$ is edge-preserving and without fixed points. Hence $G$ embeds in the graph-automorphism-group of $W$ as asserted in (v).

For $A, B$ in $W$ we have $\delta(A, B)=1$ if and only if $A, B$ are adjacent. Hence every isometry of $(W, \delta)$ is a graph-automorphism. The converse is a consequence of the definition of $\delta$. Thus (vi) holds.

Let $W$ be nice and let $S(X, W)$ denote the subset of $S(X)$ consisting of all those permutations of $X$ whose induced action on $\pi(X)$ keeps $W$ invariant. Obviously, $S(X, W)$ is a subgroup of $S(X)$. Since each singleton subset of $X$ belongs to $W$, distinct members of $S(X, W)$ induce distinct permutations of the set $W$. Also, the action of $S(X, W)$ preserves edges. Hence there is a canonical group-monomorphism from $S(X, W)$ to $\operatorname{Aut}(W)$.

LEMMA 2. With the above notation we have the following. 
(i) The canonical group-monomorphism from $S(X, W)$ to $\operatorname{Aut}(W)$ is an isomorphism.

(ii) Let $\alpha \in \operatorname{Aut}(W)$ be such that $\alpha$ leaves each $W$-orbit of $G$ invariant. Then $\alpha$ is in $G$.

Proof: Let $f$ be in Aut $(W)$. Since $f$ maps a vertex of degree 1 to another such, the collection of singleton subsets of $X$, which forms the subset of degree 1 vertices of $W$, is permuted by $f$. For each $x \in X$ the only edge ensuing from $\{x\}$ connects to $X-\{x\}$ and hence $f(X-\{x\})=X-f\{x\}$ for all $x \in X$. Let $\psi(f)$ denote the permutation of $X$ such that $\{\psi(f)(x)\}=f(\{x\})$. Consider $A, B \in W$ and let $y, z \in X$ be such that $\psi(f)(y)=z$. Then, $z \in f(A) \cap f(B)$ if and only if $\delta(X-\{z\}, f(A))=1=\delta(X-\{z\}, f(B))$ if and only if $\delta(X-\{y\}, A)=1=\delta(X-\{y\}, B)$ if and only if $y \in A \cap B$. Thus

$$
f(A) \cap f(B)=\{\psi(f)(y) \mid y \in A \cap B\} .
$$

Now $\delta(A, X-A)=1$ implies $\delta(f(A), f(X-A))=1$ that is, $X-f(A) \subseteq f(X-A)$. Since $A \cap X-A$ is empty, we must have $f(A) \cap f(X-A)=\emptyset$ and hence $X-f(A)=f(X-A)$. In other words, $f$ commutes with complementation. But then we also have

$$
f(A) \cup f(B)=\{\psi(f)(y) \mid y \in A \cup B\} .
$$

For each $A$ in $W$ we can now derive the equality

$$
f(A)=\{\psi(f)(x) \mid x \in A\}
$$

which tells us that the induced action of $\psi(f)$ coincides with that of $f$. Obviously, $\psi(f)$ is in $S(X, W)$. Let $\psi: \operatorname{Aut}(W) \rightarrow S(X, W)$ be the map sending $f$ to $\psi(f)$. Then $\psi$ is a homomorphism of groups and $\psi$ followed by the canonical monmorphism from $S(X, W)$ to $\operatorname{Aut}(W)$ is easily seen to be the identity map of $\operatorname{Aut}(W)$. This proves assertion (i). Henceforth, we identify $f$ with $\psi(f)$.

Let $K$ denote the subset of Aut $(W)$ consisting of those elements of Aut $(W)$ which leave each $W$-orbit of $G$ invariant. Clearly, $K$ is a subgroup of Aut $(W)$. Let $\alpha$ be an element of $K$. There is a $g \in G$ with $\alpha((e, 0))=g(e, 0)=(g, 0)$ where $e$ denotes the identity element of $G$. Let $\theta:=g^{-1} \alpha$. Then $\theta \in K$ and $\theta((e, 0))=(e, 0)$. Since $\{\{(g, 1)\} \mid g \in G\}$ is a $W$-orbit of $G$ it is mapped to itself by $\theta$. In particular $\theta((e, 1))$ $=(h, 1)$ for some $h \in G$. Let $E:=\{(e, 0),(e, 1)\}$. Since $E \in W$, we must have $\theta(E)=q E$ for some $q \in G$. So $\{(e, 0),(h, 1)\}=\{\theta((e, 0)), \theta((e, 1))\}=\{(q, 0),(q, 1)\}$. Hence $h=q=e$. Let $z$ be an arbitrary element of $G$ and let $A:=\{(e, 0),(z, 1)\}$, $B:=\{(z, 0),(e, 1)\}$. Again, since $A, B$ are in $W$, we have $\theta(A)=u A$ and $\theta(B)=v B$ for some $u, v \in G$. It follows that $\theta(z, 1)=(z, 1)$ and $\theta(z, 0)=(z, 0)$. In short, $\theta$ is the identity automorphism of $W$. Thus $\alpha=g \in G$ that is, $K=G$. 
We continue to use the previous notation. As above, $W$ stands for a nice subset of $\pi(X)$. Let $\mathcal{P}$ denote the set of $W$-orbits of $G$. Obviously, $\mathcal{P}$ has cardinality at least 2 . Henceforth we assume that the cardinality of $\mathcal{P}$ does not exceed that of the real numbers. Fix an injection (existence of one such is assured by our assumption)

$$
\lambda: \mathcal{P} \rightarrow[4,9 / 2]
$$

where the co-domain is a closed real interval, and an injection

$$
\omega: \mathcal{P} \rightarrow W
$$

such that $\omega(P)$ belongs to $P$ for all $P \in \mathcal{P}$ that is, choose the representatives for the $W$-orbits of $G$.

Let $V(W):=W \sqcup(G \times \mathcal{P})$. We make $V(W)$ (the vertex-set of) a graph by declaring that there is an edge between $a, b \in V$ exactly when one of the following holds:

(1) $a, b$ are in $W$ and $a \cup b=X$,

(2) $a:=(g, P)$ and $b=g \omega(P)$ with $(g, P) \in G \times \mathcal{P}$.

In view of of Lemma 1 , it follows that $V(W)$ is a connected graph of diameter at most 5 such that a vertex has degree 1 exactly when it is in $G \times \mathcal{P}$ and a vertex has degree 2 if and only if it is a degree 1 vertex in the sub-graph $W$. To simplify the notation, we let $V$ stand for $V(W)$ unless there is a need to pay attention to a specific choice of $W$.

We extend the metric $\delta$ from $W$ to the set $V$ as follows. If $a:=(g, P)$ is in $G \times \mathcal{P}$, then the unique edge ensuing from $a$ is declared to have length $\lambda(P)$. Define the distance $\delta(a, b)$ between $a, b \in V$ to be the length of the shortest edge-path (in the graph) connecting them. We also extend the action of $G$ on $W$ to an action on $V$ by declaring $g(t, P)$ $:=(g t, P)$ for all $(t, P) \in G \times \mathcal{P}$. It is straight-forward to verify that this action of $G$ preserves the edges of graph $V$. So we regard $G$ as a subgroup of Aut $(V)$, the graphautomorphism-group of $V$. The $V$-orbits of $G$ are exactly the $W$-orbits of $G$ together with the sets of the form $G \times\{P\}$. Let $\operatorname{Isom}(V, \delta)$ denote the group of isometries of the metric space $(V, \delta)$.

LEMMA 3 . The following holds.

(i) $1 \leqslant \delta\left(v_{1}, v_{2}\right) \leqslant 12$ for all $v_{1}, v_{2}$ in $V$.

(ii) $\operatorname{Isom}(V, \delta)$ is (canonically isomorphic to) $G$.

(iii) Each element of $V$ has trivial stabiliser in $G$.

PRoof: It is straightforward to verify assertions (i) and (iii). We proceed to show (ii). Let $H$ denote the isometry-group $\operatorname{Isom}(V, \delta)$. Regard $H$ as a subgroup of the group of permutations of $V$. Our identification of $H$ with $G$ rests on the following four basic observations.

(o1) The distance between any two elements of $W$ is at most 3 (see (i) of Lemma 1). 
(o2) The distance between any two (distinct) elements of $G \times \mathcal{P}$ is at least 9 .

(o3) The distance between an element of $W$ and an element of $G \times \mathcal{P}$ is at least 4 and at most $15 / 2$.

(o4) If $a:=(g, P) \in G \times \mathcal{P}$ and $b \in W$ are such that either $b$ does not belong to $P$ or $b \neq g \omega(P)$, then $\delta(a, b) \geqslant 5$.

Consider an element $\theta$ of $H$ and an element $a:=(g, P)$ of $G \times \mathcal{P}$. Since $\delta(a, g \omega(P))$ $=\lambda(P)$, we have $\delta(\theta(a), \theta(g \omega(P)))=\lambda(P)$. Note that there is a $c$ in $W$ with $\delta(g \omega(P), c)$ $=1$. From $\delta(\theta(g \omega(P)), \theta(c))=1$ it follows that $\theta(g \omega(P))$ is in $W$. Hence $\theta(a)$ has to be in $G \times \mathcal{P}$. Moreover, if $\theta(a):=(h, Q)$. then, $Q=P$ and $\theta(g \omega(P))=h \omega(P)$. In particular, $\theta$ leaves $W$ invariant and also maps each $V$-orbit of $G$ to itself.The above argument also shows that if the restriction of $\theta$ to $W$ is identity, then $\theta$ is the identity map of $V$. This way, $H$ can be regarded as a subgroup of $\operatorname{Aut}(W)$. Since $H$ leaves each $W$-orbit of $G$ invariant, in view of (ii) of Lemma 2, we have $H=G$.

Definition: Given a nice sub-set $W$ of $\pi(X)$ let $Y(G, W):=V(W)$ and correspondingly let $(Y(G, W), \delta):=(V(W), \delta)$.

REMARK. Let $G$ be finite group of order $k>2$. Then $Y\left(G, Z_{0}\right)$ has $4 k(k+2)$ points. Thus, by the above lemma, $G$ is the isometry group of a metric space of cardinality $4 k(k+2)$ and furthermore, $G$ acts properly discontinuously on this space. The reader may wish to compare this with the results of $[\mathbf{1}]$.

\section{Comb-eXtensions}

Suppose the data $(N, d, m, M, B, \partial, \beta)$ satisfies the following properties.

(p1) $M$ and $m$ are positive real numbers.

(p2) $(N, d)$ is a metric space such that $2 m<d(t, u)<2 M$ whenever $t, u$ are points of $N$ with $t \neq u$.

(p3) $(B, \partial)$ is a connected metric space of diameter $\leqslant 2 \mathrm{~m}$.

(p4) $\beta$ is a surjection from $N$ onto $B$.

Let $\operatorname{comb}(N, M, m, \beta)$ denote the quotient of $N \times[0, M]$ obtained by identifying a $(t, 0)$ with a $(u, 0)$ whenever $\beta(t)=\beta(u)$. Now we have the canonical surjection $N \times[0, M]$ $\rightarrow \operatorname{comb}(N, M, m, \beta)$. The image of $(t, a)$ via this map is denoted by $[(t, a)]$. Below we define three important mappings.

(a) $\tau: N \rightarrow \operatorname{comb}(N, M, m, \beta)$ given by $\tau(u):=[(u, M)]$.

(b) $\eta: B \rightarrow \operatorname{comb}(N, M, m, \beta)$ given by $\eta(k):=[(t, 0)]$ where $\beta(t)=k$.

(c) For points $P_{1}:=[(t, a)]$ and $P_{2}:=[(u, b)]$ of $\operatorname{comb}(N, M, m, \beta)$ define

$D\left(P_{1}, P_{2}\right):=|a-b|$ if $t=u$ and otherwise let 


$$
D\left(P_{1}, P_{2}\right):=\min \{(t, u)+2 M-a-b, a+b+\partial(\beta(t), \beta(u))\}
$$

LEMMA 4. The following holds.

(i) $\tau$ is injective.

(ii) $\eta$ is injective.

(iii) $D(\tau(u), \tau(v))=d(u, v)$ for all $u, v$ in $N$.

(iv) $D(\eta(k), \eta(l))=\partial(k, l)$ for all $k, l$ in $B$.

(v) $D$ is a metric on $\operatorname{comb}(N, M, m, \beta)$.

Proof: The needed triangle inequality for $D$ is the only non-transparent part of the above assertions. Although this inequality might be intuitively clear to an expert reader, it is helpful to sketch a verification. So, consider points $P:=[(x, a)], Q:=[(y, b)]$ and $R:=[(z, c)]$ of $\operatorname{comb}(N, M, m, \beta)$. Our proof of the inequality

$$
D(P, R) \leqslant D(P, Q)+D(Q, R)
$$

is divided into five cases.

CASE I. $x=y=z$. Follows from the triangle inequality for absolute values.

CASE II. $x=z \neq y$. In this case, we also have $x \neq y$. Interchanging $P$ and $R$ if needed, we may assume $a \geqslant c$. Since $a-c \leqslant a+b$ and $a-c \leqslant 2 M-b-c$, it suffices to consider the sub-case where $D(P, Q)=d(x, y)+2 M-(a+b)$ and $D(Q, R)=(b+c)+\partial(\beta(y), \beta(z))$. Now it suffices to note that $a-c \leqslant M$.

CASE III. $x \neq z=y$. Obviously, $x \neq y$. If

$$
a+b+\partial(\beta(x), \beta(y))>d(x, y)+2 M-(a+b),
$$

then we have

$$
d(x, y)+2 M-(a+b)+|b-c| \geqslant d(x, z)+2 M-(a+c) \geqslant D(P, R) .
$$

Otherwise, noting that $\beta(y)=\beta(z)$ and $a+b+|b-c| \geqslant a+c$ we get

$$
a+b+\partial(\beta(x), \beta(y))+|b-c| \geqslant a+c+\partial(\beta(x), \beta(z)) \geqslant D(P, R) .
$$

CASE IV. $x=y \neq z$. Obviously, $x \neq z$ and the verification is entirely similar to the one in the previous case.

CASE V. $x \neq y \neq z \neq x$. From the triangle inequality for $d$ we get

$$
d(x, y)+2 M-(a+b)+d(y, z)+2 M-(b+c) \geqslant d(x, z)+2 M-(a+c) \geqslant D(P, R) .
$$

From the triangle inequality for the metric $\partial$, we get

$$
(a+b)+\partial(\beta(x), \beta(y))+(b+c)+\partial(\beta(y), \beta(z)) \geqslant D(P, R) .
$$


From $d(x, y)>2 m \geqslant \partial(\beta(x), \beta(z))$ we deduce

$$
d(x, y)+2 M-(a+b)+(b+c)+\partial(\beta(y), \beta(z)) \geqslant D(P, R) .
$$

Likewise, from $d(y, z)>2 m \geqslant \partial(\beta(x), \beta(z))$ we deduce

$$
(a+b)+\partial(\beta(x), \beta(y))+d(y, z)+2 M-(b+c) \geqslant D(P, R) .
$$

This establishes assertion (v).

REMARKS.

1. Clearly, $(\operatorname{comb}(N, M, m, \beta), D)$ is a bounded metric space.

2. Note that for each $x \in N$ the injection $[0, M] \rightarrow \operatorname{comb}(N, M, m, \beta)$ which maps $k$ to $[(x, k)]$ identifies the interval $[0, M]$ (with its usual metric) as a metric sub-space of $(\operatorname{comb}(N, M, m, \beta), D)$.

3. In view of (i) and (iii) of the above lemma, $(N, d)$ is regarded as a metric sub-space of $(\operatorname{comb}(N, M, m, \beta), D)$.

4. Likewise, (ii) and (iv) permit us to identify the space $(B, \partial)$ as a metric sub-space of $(\operatorname{comb}(N, M, m, \beta), D)$.

LEMMA 5. Letting $E(N):=\operatorname{comb}(N, M, m, \beta)$ we have the following.

(i) If $(B, \partial)$ is arc-connected, then so is $(E(N), D) .(B, \partial)$ is a deformation retract of $(E(N), D)$. In particular, if $(B, \partial)$ is contractible, then so is $(E(N), D)$.

(ii) If $N$ is countable and card $B=1$, then $(E(N), D)$ is separable.

(iii) There is a natural group-monomorphism

$$
\rho: \operatorname{Isom}(E(N), D) \rightarrow \operatorname{Isom}(N, d) .
$$

(iv) Assume $N$ has at least 3 distinct points. Also assume that

$$
\partial(\beta(f(x)), \beta(f(y)))=\partial(\beta(x), \beta(y))
$$

for all $x, y$ in $N$ and all $f$ in $\operatorname{Isom}(N, d)$. Then, the above morphism $\rho$ is surjective, inducing an isomorphism of $\operatorname{Isom}(E(N), D)$ onto $\operatorname{Isom}(N, d)$. Moreover, the action of $\operatorname{Isom}(E(N), D)$ on $E(N) \backslash \eta(B)$ is (free and) properly discontinuous.

(v) In addition to the hypotheses of (iv) assume that for any $x$ in $N$ and any $f$ in $\operatorname{Isom}(N, d)$ whenever $\beta(f(x))=\beta(x)$, we have $f(x)=x$. Then, the stabiliser (in $\operatorname{Isom}(E(N), D))$ of a point $[(x, a)]$ is the same as the stabiliser of $x$ in $\operatorname{Isom}(N, D)$.

TERMINOLOGY. We want to draw reader's attention to our use of the term arc-connected; as in [3], an arc is meant to be a path homeomorphic to $[0,1]$.

Proof: Below we tacitly identify $\tau(N)$ with $N$ and $\eta(B)$ with $B$. Connectedness properties and assertion (ii) are evident from the construction of $E(N)$. Since

$$
h: E(N) \times[0,1] \rightarrow E(N),
$$


given by $h([(u, a)], t)=[(u, t a)]$, is easily seen to be continuous, $B$ is a deformation retract of $(E(N), D)$. Thus (i) holds.

We proceed to show the third assertion. For a positive real number $\varepsilon$, let $S_{\varepsilon}(P)$ denote the sphere of radius $\varepsilon$ with centre $P$. Given a point $P$ of $E(N)$ we note the following.

(1) $S_{\varepsilon}(P)$ is singleton for all sufficiently small $\varepsilon>0$ if and only if $P$ is in $N$.

(2) $S_{m}(P)$ is doubleton for all sufficiently small $\varepsilon>0$ if and only if $P$ is not in $N \cup B$.

Property (1) ensures that each isometry of $(E(N), D)$ maps $N$ to itself and hence upon restriction to $N$ yields an isometry of $(N, d)$. Likewise, property (2) ensures that $B$ and (hence) the complement of $N \cup B$ are invariant under the action of $\operatorname{Isom}(E(N), D)$. Let $\rho: \operatorname{Isom}(E(N), D) \rightarrow \operatorname{Isom}(N, d)$ denote the 'restriction-to- $N$ ' map. Clearly $\rho$ is a homomorphism of groups. Let $\theta$ be in the kernel of $\rho$. Consider a point $P:=[(u, a)]$ and suppose $\theta(P):=[(t, b)]$. As observed earlier, $\theta$ maps $Q:=[(u, 0)]$ to some $[(v, 0)]$. Comparing the distance of each of these from the ( $\theta$-fixed ) point $[(u, M)]$ we at once see that $[(v, 0)]=Q$. Suppose $a \neq 0$. Now equalities $D(P, Q)=D([(t, b)], Q)$ and $D(P,[(u, M)])=D([(t, b)],[(u, M)])$ readily imply

$$
d(u, t)=b-a=-\partial(\beta(u), \beta(t)) .
$$

Needless to say that the above holds only when $d(u, t)=0=b-a$ that is, $\theta(P)=P$. This proves the injectivity of $\rho$ as claimed in (iii).

With the hypotheses of (iv), given an isometry $f$ of $(N, d)$ the prescription $f([(u, a)]$ $:=[(f(u), a)]$ yields a well-defined isometry of $(E(N), D)$. Thus $\rho$ is surjective and consequently it is an isomorphism. Identify $\operatorname{Isom}(E(N), D)$ with $\operatorname{Isom}(N, d)$. Suppose an isometry $f$ fixes $[(x, a)]$. If $a \neq 0$, then we must have $f(x)=x$. If $a=0$, then we must have $\beta(f(x))=\beta(x)$. The additional hypothesis in (v) ensures that we have $f(x)=x$ even when $a=0$. The nature of $D$ makes it easy to see that the action of $\operatorname{Isom}(N, d)$ on $E(N) \backslash B$ is properly discontinuous.

REMARKS.

1. Note that the assumed bounded-ness of $(B, \partial)$ is used only in establishing the triangle inequality for $D$. Without assuming this boundedness, does there exist a metric on $E(N)$ for which our Lemma 5 remains valid?

2. Let $P_{1}:=[(t, a)]$ and $P_{2}:=[(u, b)]$ be points of $E(N)$. Define

$$
D^{*}\left(P_{1}, P_{2}\right):= \begin{cases}|a-b| & \text { if } t=u, \\ a+b+\partial(\beta(t), \beta(u)) & \text { if } t \neq u .\end{cases}
$$

Then $D, D^{*}$ are equivalent metrics on $E(N)$ (verification left to the reader). 
In the remaining part of this section we restrict our attention to the case where $B$ is the trivial metric space consisting of a single point. Without any loss, we assume $M=1$. Since we want to focus only on the topology of $(E(N), D)$, in view of the above remark it is permissible to replace $D$ by its equivalent $D^{*}$ and then we are free to ignore the metric on $N$. To simplify the notation, let $X:=E(N)$, let $a \dot{t}:=[(t, a)]$, let $O:=[(t, 0)]$ and let

$$
\|a \dot{u}-b \dot{v}\|:=D^{*}([(u, a)],[(v, b)]) .
$$

Likewise, a point $\left(a_{1} \dot{x}_{1}, \cdots, a_{n} \dot{x}_{n}\right)$ of $X^{n}$ is denoted by $a \dot{x}$ where $a$ stands for $\left(a_{1}, \cdots, a_{n}\right)$ and $x$ stands for $\left(x_{1}, \cdots, x_{n}\right)$. It is tacitly assumed that the metric on $X^{n}$ is the one given by

$$
\|a \dot{x}-b \dot{y}\|:=\left\|a_{1} \dot{x}_{1}-b_{1} \dot{y}_{1}\right\|+\cdots+\left\|a_{n} \dot{x}_{n}-b_{n} \dot{y}_{n}\right\| .
$$

Henceforth by $X^{n}$ we mean the metric space $\left(X^{n},\|\|\right)$. Observe that the natural projection $p: X^{n} \rightarrow[0,1]^{n}$ is a weak contraction that is, it does not increase distances. Multiplication of tuples in $[0,1]^{n}$ is meant to be coordinatewise.

Lemma 6 .

(i) Let $\mu: X^{n} \rightarrow(0,1] \cup\{-\infty\}$ map $P:=a \dot{x}$ to the minimum of $\left\{a_{1}, \cdots, a_{n}\right\} \backslash\{0\}$ (by convention, $-\infty$ is the minimum of the empty set). Suppose $Q:=b \dot{y}$ is a point of $X^{n}$ at distance at most $\mu(P)$ from $P$. If $r, s$ are elements of $[0,1]^{n}$, then

$$
\|(r a) \dot{x}-(s b) \dot{y}\|=\left|r_{1} a_{1}-s_{1} b_{1}\right|+\cdots+\left|r_{n} a_{n}-s_{n} b_{n}\right| .
$$

(ii) Let $\phi$ be a continuous function from $[0,1]^{m} \times U$ to $[0,1]^{n}$ where $U$ is an open subset of $[0,1]^{n}$ not containing the zero vector. Then the (corresponding) function

$$
f:[0,1]^{m} \times p^{-1}(U) \rightarrow X^{n}
$$

given by $f(t, a \dot{x}):=(\phi(t, a) a) \dot{x}$ is continuous.

Proof: Consider points $P, Q$ as in (i). If there is an $1 \leqslant j \leqslant n$ such that $a_{j} b_{j} \neq 0$ and $x_{j} \neq y_{j}$, then $\|a \dot{x}-b \dot{y}\| \geqslant a_{j}+b_{j}>\mu(P)$. Thus for all $1 \leqslant j \leqslant n$

$$
\left\|\left(r_{j} a_{j}\right) \dot{x}_{j}-\left(s_{j} b_{j}\right) \dot{y}_{j}\right\|=\left|r_{j} a_{j}-s_{j} b_{j}\right|
$$

and (i) follows.

To prove (ii) consider a point $(t, P)$ where $P:=a \dot{x}$ and a positive real number $\varepsilon$. Our hypothesis ensures that $\mu(P)>0$. Using continuity of the map

$$
(t, c) \rightarrow \phi(t, c) c=:\left(\phi_{1}(t, c) c_{1}, \cdots, \phi_{n}(t, c) c_{n}\right)
$$

find $\theta>0$ such that

$$
\left|\phi_{1}(t, a) a_{1}-\phi_{1}(s, b) b_{1}\right|+\cdots+\left|\phi_{n}(t, a) a_{n}-\phi_{n}(s, b) b_{n}\right|<\varepsilon
$$


for all $(s, b)$ with

$$
\left|t_{1}-s_{1}\right|+\cdots+\left|t_{m}-s_{m}\right|+\left|a_{1}-b_{1}\right|+\cdots+\left|a_{n}-b_{n}\right|<\theta .
$$

Now continuity of $f$ is readily established by choosing $\delta:=\min \{\theta, \mu(P)\}$.

Definitions: Define sub-spaces $S^{2}(X)$ and $Y$ of $X^{3}$ by

$$
\begin{aligned}
S^{2}(X) & :=\{(a \dot{x}, b \dot{y}, c \dot{z}) \mid a+b+c=1\}, \\
Y: & :=\{(a \dot{x}, b \dot{y}, O) \mid a+b=1\} .
\end{aligned}
$$

For each $z \in N$ we define a metric subspace $Y(z)$ of $X^{3}$ by

$$
Y(z):=\left\{(a \dot{x}, b \dot{y},(1-a-b) \dot{z}) \mid(a \dot{x}, b \dot{y}) \in X^{2} \text { and } a+b \leqslant 1\right\}
$$

\section{REMARKS.}

1. Earlier we have used the notation $S(X)$ to denote the group of permutations of $X$. Obviously, $S^{2}(X)$ bears no relation to $S(X)$.

2. Since the topology of $(N, d)$ is discrete, after having ignored the metric $d$ cardinality of $N$ is all that matters. More precisely, let $N^{*}$ be a non-empty set whose cardinality equals that of $N$ and let $d^{*}$ be a metric on $N^{*}$ such that $0<2 m^{*}<d^{*}(t, u)<2 M^{*}$ for all distinct $t, u \in N^{*}$. Then $(X,\|\|)$ is homeomorphic to $\left(E\left(N^{*}\right), D\right)$ where the later space is the comb-extension of $\left(N^{*}, d^{*}\right)$ with trivial base $B$ as indicated before. Thus it is more suggestive to denote $X$ by $X_{k}$ and $S^{2}(X)$ by $\Sigma_{k}$ where $k$ is the cardinality of $N$.

3. If $N$ is countable, then $X$ can be topologically embedded in $\mathbb{R}^{2}$ and hence $X^{n}, S^{2}(X)$ are homeomorphic to subspaces of $\mathbb{R}^{2 n}, \mathbb{R}^{6}$ respectively.

LEMMA 7. The following holds.

(i) $S^{2}(X)$ is a polyhedral surface.

(ii) $S^{2}(X)$ is a union of sets $Y(t)$ as $t$ ranges over $N$.

(iii) $Y$ is arc-connected and $Y(u) \cap Y(v)=Y$. for all $u \neq v$ in $N$.

(iv) $S^{2}(X)$ is a deformation retract of $X^{3} \backslash\{(O, O, O)\}$.

(v) $Y(z)$ is homeomorphic to a cone over $Y$. In particular, each $Y(z)$ is contractible.

(vi) $Y(u) \cup Y(v)$ is simply-connected for all $u \neq v$ in $N$.

PROOF: For each $(x, y, z) \in N^{3}$ let $I(x, y, z)$ denote the subspace $\{(a \dot{x}, b \dot{y}, c \dot{z})\}$ of $X^{3}$. Note that each $I(x, y, z)$ is a closed subset homeomorphic to the standard cube and $X^{3}$ is a union of these cubes as $(x, y, z)$ ranges over $N^{3}$. Moreover, any two of these cubes meet along a face (edge, vertex) containing $(O, O, O)$.

Since $S^{2}(X) \cap I(x, y, z)$ is just the standard 2-simplex, it follows that $S^{2}(X)$ is a connected simplicial complex of pure dimension 2 , that is, a polyhedral surface as asserted in (i). Verification of (ii) and (iii) is straightforward. 
Let $U:=[0,1]^{3}-\{(0,0,0)\}$ and $\phi:[0,1] \times U \rightarrow[0,1]^{3}$ be the continuous function defined by

$$
\phi(t,(a, b, c)):=\left(1-t+\frac{t}{a+b+c}, 1-t+\frac{t}{a+b+c}, 1-t+\frac{t}{a+b+c}\right) .
$$

In view of (ii) of Lemma 6 it follows that the corresponding map

$$
\begin{gathered}
F:[0,1] \times\left(X^{3}-\{(O, O, O)\}\right) \rightarrow X^{3}-\{(O, O, O)\} \\
F(t, \alpha \dot{v}):=(\phi(t, \alpha) \alpha) \dot{v}
\end{gathered}
$$

is a (strong) deformation retraction of $X^{3} \backslash\{(O, O, O)\}$ to $S^{2}(X)$.

Using (ii) of Lemma 6 it is easy to verify that the map $Y \times[0,1] \rightarrow Y(z)$ given by

$$
((a \dot{x}, b \dot{y}, \dot{O}), t) \rightarrow((t a) \dot{x},(t b) \dot{y},(1-t) \dot{z})
$$

is continuous and consequently $Y(z)$ is a cone over $Y$.

Finally, observe that if $u, v$ are distinct, then $Y(u) \cup Y(v)$ is just a double-cone (that is, a suspension) over the arc-connected space $Y$ and hence it is indeed simplyconnected.

Acknowledgement. The author is indebted to N. Brodskiy for bringing observations (ii), (iii) and (iv) of Lemma 7 to his notice.

LEMma 8. $S^{2}(X)$ is simply-connected.

Proof: Fix a point $z$ of $N$. By $P_{u}$ we mean the vertex point $(O, O, u)$ of the cone $Y(u)$. If $r$ is a real number in the interval $[0,1]$ and $u$ is in $N$, then let

$$
Y(r, u):=\left\{Q \in Y(u) \mid\left\|Q-P_{u}\right\|>r\right\}
$$

Let $\Gamma$ be the set of all functions from $N$ to $[0,1]$ which are constant outside a finite subset of $N$. Let $I:=\Gamma \times N$. For an element $(\rho, u)$ of $I$ let

$$
Z(\rho, u):=\left[\cup_{t \in N} Y(\rho(t), t)\right] \cup Y(u) \cup Y(z) .
$$

Observe that

(i) $S^{2}(X)=\bigcup_{(\rho, u) \in I} Z(\rho, u)$,

(ii) $Z\left(\tau_{1}, u_{1}\right) \cap \cdots \cap Z\left(\tau_{n}, u_{n}\right)$ equals $Z(\theta, w)$ for some $(\theta, w)$ in $I$,

(iii) $Z(\rho, u)$ is an open, arc-connected subset of $S^{2}(X)$ and

(iv) $(O, O, z)=P_{z}$ is in $\cap_{(\rho, u) \in I} Z(\rho, u)$.

Further, it is straightforward to verify that $Y(u) \cup Y(z)$ is a deformation retract of $Z(\rho, u)$ and hence, in view of (vi) of Lemma $7, Z(\rho, u)$ is simply-connected for all $(\rho, u)$ in $I$. Applying the generalised van Kampen theorem (see [2]) we conclude that $\left.\pi_{1}\left(S^{2}(X), P_{z}\right)\right)=1$. 


\section{Main TheOREM}

Definitions: Let $G$ be a group and $(M, d)$ be a metric space. By an isometric action of $G$ on $(M, d)$ we mean a group-homomorphism $\alpha$ from $G$ to $\operatorname{Isom}(M, d)$. Given an isometric action $\alpha$ of $G$ on $(M, d)$, by a $(G, \alpha)$-extension of $(M, d)$ we mean a metric space $\left(M^{*}, d^{*}\right)$ together with a $\phi: G \rightarrow H:=\operatorname{Isom}\left(M^{*}, d^{*}\right)$ satisfying the following properties.

(1) $(M, d)$ is an $H$-invariant metric subspace of $\left(M^{*}, d^{*}\right)$.

(2) $\phi$ is a group-isomorphism of $G$ onto $H$.

(3) Letting $h: H \rightarrow \operatorname{Isom}(M, d)$ denote the 'restriction-to- $M$ ' map we have $\alpha=h \circ \phi$.

(4) Via $\phi$, the group $G$ acts (freely,) properly discontinuously on the subspace $M^{*} \backslash M$.

(5) $(M, d)$ is a deformation retract of $\left(M^{*}, d^{*}\right)$.

LEMMA 9. Let $G$ be a nontrivial group. of cardinality not exceeding $2^{N_{0}}$. Then, there exists an (arc-connected) contractible, bounded metric space $(\mathcal{M}, \mathcal{D})$ with a marked point $O$ such that

(i) $G$ is isomorphic to $\operatorname{Isom}(\mathcal{M}, \mathcal{D})$,

(ii) $G$ acts properly discontinuously on $\mathcal{M} \backslash\{O\}$ and

(iii) if $G$ is countable, then $(\mathcal{M}, \mathcal{D})$ is separable.

Proof: Let $(B, \partial)$ be the trivial metric space, that is, where $B$ has only one point $O$. Let $(N, d)$ be either the space $\left(Y\left(G, Z_{0}\right), \delta\right)$ or any one of $\left(Y\left(G, Z_{r}\right), \delta\right)$ where $3 \leqslant r \leqslant \infty$. Let $\beta$ be the obvious surjection and let $(\mathcal{M}, \mathcal{D})$ be the $\operatorname{space}(\operatorname{comb}(N, 10,1 / 10, \beta), D)$ (our choices of $M$ and $m$ are permissible in view of Lemma 3). Since $\operatorname{Isom}(N, d)$ is $G$ by Lemma 3, assertion (i) follows from (iv) of Lemma 5 . Note that $s t a b_{G}(x)$ is trivial for each $x$ in $N$ (as ensured by (iii) of Lemma 3) and thus (ii) follows from Lemma 5 . Assertion (iii) follows by noting that when $G$ is countable, $N$ is countable.

LEMMA 10. Let $G$ be an infinite group with $\operatorname{card}(G) \leqslant 2^{\aleph_{0}}$. Let $\alpha$ be an isometric action of $G$ on a connected, bounded metric space $(B, \partial)$ such that $\operatorname{card}(B) \leqslant 2^{\kappa_{0}}$. Then, there exists a $(G, \alpha)$-extension $(\mathcal{M}, \mathcal{D})$ of $(B, \partial)$ such that the following holds.

(i) $(\mathcal{M}, \mathcal{D})$ is a bounded metric space.

(ii) If $(B, \partial)$ is arc-connected, then so is $(\mathcal{M}, \mathcal{D})$.

(iii) If $(B, \partial)$ is contractible, then so is $(\mathcal{M}, \mathcal{D})$.

(iv) If $G$ is countable and card $B=1$, then $(\mathcal{M}, \mathcal{D})$ is separable.

Proof: If $G$ is countable and $B$ is uncountable, then let $N=Y(G, Z)$. Otherwise, let $N=Y\left(G, Z_{0}\right)$ (or any one of $Y\left(G, Z_{\mathrm{r}}\right)$ ). Scale the metric $\delta$ (appearing in Lemma $3)$ to obtain a metric $d$ on $N$ such that for every pair $(t, u)$ of distinct points of $N$ the 
distance $d(t, u)$ is strictly greater than the diameter of $(B, \partial)$. It is important to observe that, in view of Lemma 3 , we have $G=\operatorname{Isom}(N, d)$ and $G$ acts properly discontinuously on $(N, d)$. Fix a set of representatives $T$ for the $N$-orbits of $G$. Without loss we may identify $N$ with $G \times T$. In this form the action of $G$ on $N$ is simply left-multiplication in the first coordinate. The remarks immediately following our description of the sets $Z, Z_{r}$ show that $\operatorname{card} T \geqslant \operatorname{card} B$. Fix a set of representatives $S$ for the $B$-orbits of $G$. Choose a surjection $\psi: T \rightarrow S$ and let $\beta: N \rightarrow B$ be the surjection defined by : $\beta(x)=g \psi(t)$ if $x=(g, t)$. Since $\alpha$ is an isometric action, the requirements of assertion (iv) of Lemma 5 are satisfied. Choose positive real numbers $M, m$ so that the diameter of $(B, \partial)$ is at most $2 m$ and we have $2 m<d(t, u)<2 M$ for all pairs $(t, u)$ of distinct points of $N$. Now let $(\mathcal{M}, \mathcal{D})$ be the space $(\operatorname{comb}(N, M, m, \beta), D)$. Then, our assertions follow from Lemma 5 in a straightforward manner.

THEOREM. Given a cardinal $k \geqslant 2$ there exists a simply-connected polyhedral surface $\Sigma_{k}$ endowed with a bounded metric $d_{k}$, such that the following holds.

(i) Each group $G$ of cardinality $k$ has an isometric, properly discontinuous action on $\left(\Sigma_{k}, d_{k}\right)$. Moreover, if $k \leqslant \aleph_{0}$, then $\left(\Sigma_{k}, d_{k}\right)$ is a separable metric space.

(ii) If $\aleph_{0} \leqslant k \leqslant 2^{\aleph_{0}}$ and $G$ is a group of cardinality $k$, then $\Sigma_{k}$ has a (simplyconnected) extension $M_{G}$ carrying a bounded metric $d_{G}$ such that the action of $G$ extends to a properly discontinuous action on $\left(M_{G}, d_{G}\right)$, the restriction of $d_{G}$ to $\Sigma_{k}$ is equivalent to $d_{k}$ and $G$ is (isomorphic to) the full isometry-group of $\left(M_{G}, d_{G}\right)$.

Proof: To prove (i) let $(B, \partial)$ be the 1-point (trivial) metric space, let $N=G$ (with discrete topology) and let $X=E(N)$ be the metric space as in the last part of the previous section. Clearly, $X$ is a cone on $G$. Left-multiplication by elements of $G$ naturally extends to an isometric action on $X$ having the vertex as the only fixed point. The induced coordinatewise action of $G$ on $X^{3}$ keeps $S^{2}(X)$ invariant. Thus we get a properly discontinuous, isometric action of $G$ on $\left(S^{2}(X),\|\|\right)$. By the remarks preceding Lemma 7 , the space $\left(S^{2}(X),\|\|\right)$ depends only on the cardinality of $N$. Hence employing $\left(\Sigma_{k}, d_{k}\right)$ to denote $\left(S^{2}(X),\|\|\right)$ is indeed well justified. Note that when $G$ is countable, $X$ is a separable metric space.

To prove (ii) we start with the one-point metric space $(B, \partial)$ and construct $(\mathcal{M}, \mathcal{D})$ exactly as in Lemma 9. The choice of $N$ as in Lemma 9 ensures that cardinality of $N$ equals $k$ (see the remarks preceding Lemma 1). Let $d_{G}$ be the (product) metric on $S^{2}(M)$ induced by $D$. By Lemma $9, d_{G}$ is a bounded metric. As above, in view of the last part of the previous section, $S^{2}(M)$ is naturally identified with $\Sigma_{k}$ and then $d_{G}$ is equivalent to $d_{k}$. Action of $G$ on $\mathcal{M}$ extends naturally to a coordinatewise action on $\mathcal{M}^{3}$. Obviously $S^{2}(M)=\Sigma_{k}$ is invariant under this action. Hence, by (i) and (ii) of Lemma $9, G$ has an isometric, properly discontinuous action on the metric space $\left(\Sigma_{k}, d_{G}\right)$. Let $\alpha$ denote this action. Starting from $(B, \partial):=\left(\Sigma_{k}, d_{G}\right)$ we construct a $(G, \alpha)$-extension $\left(M_{G}, d_{G}\right)$ of $(B, \partial)$ as in Lemma 10 . Then $\left(\Sigma_{k}, d_{G}\right)$ is a deformation retract of $\left(M_{G}, d_{G}\right)$. In particular, 
$\left(M_{G}, d_{G}\right)$ is simply-connected. Also, $G$ is the full group of isometries of $\left(M_{G}, d_{G}\right)$ acting properly discontinuously on $\left(M_{G}, d_{G}\right)$.

REMARK. As a consequence of (i), the orbit space $\Sigma_{k} / G$ has fundamental group $G$. Although it is well known that for any group $G$ there is an Eilenberg-MacLane space having $G$ as its fundamental group (see [4]), the above construction is much simpler.

\section{Questions}

Here we formulate some questions which are natural variations of the above result. In the following assume $G$ to be a group whose cardinality does not exceed that of the real numbers.

1. Does there exist a contractible, separable (bounded) metric space on which $G$ has a properly discontinuous, isometric action?

2. Does there exist a contractible, (bounded) metric subspace of some $\mathbb{R}^{n}$ having $G$ as the (full) group of isometries?

3. Do either of the above two questions have an affirmative answer if $G$ is countable?

4. Assuming $G$ to be finitely generated, does there exist a contractible, separable (bounded) metric space on which $G$ acts isometrically and such that the isotropy groups are finite groups of uniformly bounded order?

It seems rather unlikely that an arbitrary countable group $G$ can be realised as the full group of isometries of a contractible metric subspace of some $\mathbb{R}^{n}$.

\section{REFERENCES}

[1] D. Asimov, 'Finite groups as isometry groups', Trans. Amer. Math. Soc. 216 (1976), 389- 391.

[2] R.H. Crowell, 'On the van Kampen theorem', Pacific J. Math. 9 (1959), 43-50.

[3] M.C. Gemignani, Elementary topology (Dover Publications, New York, 1990).

[4] E.H. Spanier, Algebraic topology (McGraw-Hill Inc., New York, Toronto, London, 1966).

Department of Mathematics

The University of Tennessee

Knoxville, TN 37996-1300

United States of America

e-mail: mulay@math.utk.edu 